\title{
(2) OPEN ACCESS \\ Common infections, mental health problems and healthcare use in people with inflammatory bowel disease: a cohort study protocol
}

\author{
Peter Irving, ${ }^{1,2}$ Kevin Barrett, ${ }^{3}$ Daniel Tang, ${ }^{4}$ Monica Nijher, ${ }^{4}$ Simon de Lusignan (D) ${ }^{5,6}$
}

\section{${ }^{1}$ Department of \\ Gastroenterology, Guy's and \\ Saint Thomas' NHS Foundation \\ Trust, London, UK \\ ${ }^{2} S c h o o l$ of Immunology and \\ Microbial Sciences, King's \\ College London, London, UK \\ ${ }^{3}$ New Road Surgery, Croxley \\ Green, UK \\ ${ }^{4}$ Pfizer Ltd, Tadworth, UK \\ ${ }^{5}$ Nuffield Department of Primary \\ Care Health Sciences, University \\ of Oxford, Oxford, UK \\ ${ }^{6}$ Royal College of General \\ Practitioners (RCGP) Research \\ and Surveillance Centre (RSC), \\ London, United Kingdom}

Correspondence to

Professor Simon de Lusignan, Nuffield Department of Primary Care Health Sciences, University of Oxford, Oxford, UK; simon.

delusignan@phc.ox.ac.uk

Published Online First

17 September 2020

\begin{abstract}
Introduction People with inflammatory bowel disease (IBD) are at increased risk of pneumonia and herpes zoster, yet other common infection types have not been explored. Anxiety and depression are more prevalent in IBD; however, the impact of these conditions on primary care healthcare use in IBD is not known.

Methods and analysis We will perform two retrospective studies using a large English populationbased primary care cohort to compare the following outcomes in people with IBD and matched controls: incident infections (Study 1) and prevalent mental health problems and healthcare use, overall and in those with and without mental health problems (Study 2). All adults registered with general practices contributing to Royal College of General Practitioners Research and Surveillance Centre database between 1 January 2014 and 1 January 2019 are eligible. Infection outcomes comprise the incidence of common infections (upper respiratory tract infections, pneumonia, acute bronchitis, influenza and influenza-like illnesses, skin infections, herpes simplex and herpes zoster infections, genital infections, urinary tract infections and gastrointestinal infections) and any viral infection. Mental health and healthcare use outcomes are: prevalence of depressive episodes; anxiety episodes; recurrent depression; rates of primary care and emergency secondary care visits; primary-care issued sick notes (reflecting time off work). Analyses will be adjusted for sociodemographic factors recorded in the primary care record.

Discussion These studies will quantify the infection risk in IBD, the excess burden of anxiety and depression in a population-based IBD cohort, and the impact of mental health conditions on healthcare use and time off work. Greater understanding and awareness of infection risk and common mental health issues will benefit people with IBD and healthcare practitioners and will guide policy makers as allocation of resource may be guided by the real-world information produced by these studies. Trial registration number NCT03836612.
\end{abstract}

\section{INTRODUCTION}

The inflammatory bowel diseases (IBD), ulcerative colitis (UC) and Crohn's disease (CD) are chronic inflammatory disorders primarily affecting the gastrointestinal (GI) tract and characterised by a fluctuating or continuous course of symptoms such as abdominal pain, bloody diarrhoea, lack of appetite, weight loss and fatigue.

The aetiology of IBD remains incompletely understood, but its pathogenesis is associated with dysregulated immune responses that drives a persistent inflammatory state within the intestinal mucosa. CD and UC have specific clinical and histological features: ${ }^{1} \mathrm{CD}$ is characterised by transmural inflammation that can affect the entire GI tract, from mouth to anus, usually involving all layers of the gut wall, with a discontinuous and patchy appearance of affected areas. ${ }^{2}$ UC affects the most superficial mucosal layer of the gut wall, usually arising from the rectum and extending proximally along the colon, potentially along its entire length. ${ }^{3}$ The major goals of therapy in IBD are to induce remission and to modify the disease course leading to improved quality of life and minimal disease-related disability. This can require a range of medication classes including anti-inflammatory, immunomodulating and biological medications. ${ }^{4}$

\section{Infections in inflammatory bowel disease}

Many therapies used for the treatment of IBD work through modification of the activity of the immune system; it is, therefore, perhaps unsurprising that many drugs used to treat IBD are associated with an increased risk of infection with both common and atypical pathogens. ${ }^{5}$ As well as drug therapy, malnutrition, surgery and increased pathogen exposure (through, for example, the hospital environment) may comprise additional infection risk factors in IBD. ${ }^{5}$

An increased risk for pneumonia and herpes zoster among people with IBD versus controls has been reported: in an observational study of patients with CD ( $\mathrm{n}=50$ 932), UC $(\mathrm{n}=56$ 403) and unspecified IBD $(n=1269)$, patients with CD and UC were at increased risk of pneumonia versus matched controls and the use of biological medications and systemic corticosteroids was associated with additional risk. ${ }^{6}$ Another observational study of people with CD $(n=7823)$ and UC $(n=11930)$ found a greater incidence of herpes zoster compared with matched controls, and corticosteroids and azathioprine/6-mercaptopurine were also significantly associated with additional risk. ${ }^{7}$

As well as pneumonia and herpes zoster, the risk of opportunistic infections has been reported to increase in patients with IBD versus matched controls. ${ }^{6}$ In a study of 100 patients with IBD vs 200 matched controls, the risk of opportunistic infection in IBD increased with corticosteroids (OR, 3.4; 95\% CI 1.8 to 6.2), azathioprine/6mercaptopurine (OR, 3.1; 95\% CI 1.7 to 5.5 ) and infliximab (OR, 4.4; 95\% CI 1.2 to 17.1$){ }^{6}$ In 
a meta-analysis of randomised trials in IBD, among 4135 and 2919 patients who received antitumour necrosis factor alpha $(\mathrm{TNF} \alpha)$ therapy and placebo, respectively, the rate of opportunistic infection for anti-TNF $\alpha$ therapy versus placebo was 39 $(0.9 \%)$ and $9(0.3 \%)$, respectively. ${ }^{8}$ The relative risk of tuberculosis for anti-TNF $\alpha$ therapy versus placebo was 2.52 (95\% CI 0.62 to 10.21 ) and other infections that occurred in the antiTNF $\alpha$ group were Mycobacterium tuberculosis $(n=8)$, herpes simplex infection $(n=8)$, oral or oesophageal candidiasis $(n=6)$, herpes zoster infection $(n=6)$, varicella-zoster virus infection $(n=2)$, cytomegalovirus or Epstein-Barr virus infection $(n=2)$ and Nocardia infection $(n=1){ }^{8}$ It has been suggested that an increased prevalence of neutropenia or lymphopaenia in IBD could increase infection risk but previous studies have not shown a clear association with opportunistic infections. ${ }^{6}{ }^{9}$ It remains unclear whether neutropenia or lymphopaenia are more common in IBD and whether they are a major contributor to infection risk.

The existing data suggest a substantially increased burden of infections in IBD; however, evidence is largely limited to selected secondary or tertiary care cohorts or clinical trials. The risk of pneumonia, herpes zoster and opportunistic/uncommon infections has been examined but other common infection types have not been well explored. Furthermore, the overall burden of common infections in an unselected population-based cohort of people with IBD is unknown.

\section{Mental health in inflammatory bowel disease}

The prevalence of depression and anxiety are reported to be higher in people with IBD compared with controls. ${ }^{10}$ In a recent population-based study in the USA, among 12540 patients with IBD aged 5-24 years, the prevalence of psychiatric disorders was $21.6 \%$, compared with $6 \%$ among controls. ${ }^{11}$ In a systematic review of $\sim 150000$ patients with IBD, the pooled prevalence of depression and anxiety symptoms was $15.2 \%$ and $20.5 \%$, respectively, with higher rates of both observed in $C D$ versus UC. ${ }^{10}$ In a population-based survey in the USA, the prevalence of depression was $16 \%$, resulting in a mean annual IBD-related increase in healthcare costs of \$17 706 compared with patients with IBD without depression. ${ }^{12}$ However, the majority of existing studies exploring the prevalence of psychiatric conditions in IBD have used selected secondary care populations, patient-reported symptoms as a surrogate marker or non-validated definitions of mental health conditions. Data on the prevalence of mental health conditions using validated definitions in a large unselected IBD cohort are currently lacking.

\section{Burden and costs of inflammatory bowel disease}

IBD is often a life-long condition requiring both medical and surgical intervention which can have negative effects on relationships, life activities and mental well-being. Many patients with IBD experience fluctuating and unpredictable symptoms that require multidisciplinary care and whereas many patients maintain long-term employment after diagnosis, IBD can be associated with unemployment, greater sick-leave and decreased work productivity. ${ }^{13}$ Several studies have reported the substantial direct and indirect costs of IBD,${ }^{14}$ including a population-based study in Denmark which showed that among 513 patients with IBD between 2004 and 2015, the total cost of IBD was $€ 42.6$ million, which was mainly driven by hospitalisation. ${ }^{15}$ IBD, therefore, represents a substantial burden to those affected, as well as having economic ramifications and a substantial healthcare burden. However, robust data are lacking for healthcare use and the impact of psychiatric illness on healthcare use among patients with IBD.

\section{Aims}

We aim to perform two retrospective studies using a large population-based cohort in England to evaluate outcomes in patients with IBD compared with matched controls:

1. Describe the incidence of a wide range of common infections in IBD and controls (Study 1).

2. Describe the relationships between incidence of infection in IBD and sociodemographic factors, medication use and time-varying blood count parameters (eg, neutrophil count) (Study 1).

3. Describe the prevalence of mental health problems (comprising anxiety episodes, depressive episodes and recurrent depressive disorder) in IBD and controls (Study 2).

4. Describe the relationship between these mental health conditions in IBD and healthcare use (Study 2).

\section{METHODS AND ANALYSIS \\ Data source}

The Royal College of General Practitioners (RCGP) Research and Surveillance Centre (RSC) database will be used, which comprises the pseudonymised primary care records of individuals registered with a large network of general practices. It provides a broadly representative sample of the primary care population in England. ${ }^{16}$ The RCGP RSC database contains data from a registered population of 2 million people, with information on clinical diagnoses, anthropometric measurements (eg, body mass index (BMI)), laboratory tests (eg, neutrophil count) and prescriptions, coded with the Read coding system (a thesaurus of clinical terms) .

UK primary care has a registration-based system (each patient registers with one general practice) and has been computerised since the 1990s, with electronic laboratory links and payfor-performance data available from 2004 that has resulted in consistent, high-quality clinical data entry about chronic disease. ${ }^{17}$ Studies and outcomes based on RCGP RSC data have been published across multiple chronic diseases. ${ }^{18} 19$

The RCGP RSC is also the primary infectious disease sentinel network for the UK, providing a weekly infections data return since 1964; it is used to monitor trends in infectious disease and investigate real-world vaccine efficacy. ${ }^{16}$ General practices within the network are provided with feedback on their coding of infectious diseases. A particular emphasis of this feedback mechanism is 'episode typing', which designates cases as first, new or ongoing, thereby helping to differentiate incidence from prevalence. ${ }^{20}$ The resulting data quality within the database means it provides a world-leading dataset for infection research. ${ }^{16}$

\section{Population}

For the infections study (Study 1 ), all adults (aged $\geq 18$ ) registered with a general practice for $\geq 1$ year and contributing to the RCGP RSC between 1 January 2014 and 1 January 2019, are eligible for inclusion. For the mental health study (Study 2), we will examine the most recent 3 -year period (1 January 2016 to 1 January 2019). The longer time period for Study 1 is to ensure we maximise capture of rarer infections in the analysis. To minimise the impact of 'ghost' patients, each patient must have $\geq 1$ consultation over the study period. 


\section{Exposed cohort with IBD}

All individuals with an existing or incident diagnosis of UC or $\mathrm{CD}$ over the study period are eligible for inclusion in the IBD cohort. The use of diagnostic codes to identify IBD from UK primary care records has been validated and shown to differentiate between UC and CD. ${ }^{21}$ The Read codes used to identify UC and $C D$ will be based on a validated codes list ${ }^{22}$ and mapped to both Read code versions used within the RCGP RSC network (online supplementary file 1). The use of diagnostic codes to identify IBD has also been shown to have good validity in US claims databases. ${ }^{23}$

Start of follow-up for an individual with IBD is defined as the latest of the study start date (if the individual has an existing diagnosis prior to the study period) or the date of diagnosis of IBD (if the individual has an incident diagnosis during the study period).

\section{Matched unexposed cohort}

Separately for both studies, individuals with IBD will be matched at their index date (the start of follow-up date) with four unexposed individuals at general practice level by current age, sex and time since practice registration (nearest neighbour matching, with replacement). The eligible pool of unexposed individuals at each index date will include individuals actively registered at that date with no history of IBD and at $\geq 1$ year of follow-up in RCGP RSC. Follow-up for each matched control will start on the index date of their matched case with IBD. Those diagnosed with IBD after the study start date will be included in the pool of eligible unexposed population, but if matched, will be excluded from the study from the date of their diagnosis.

\section{End of follow-up}

The end of follow-up for both studies will be defined as the earliest of: the study end-date (1 January 2019), the date of patient transfer from an included general practice, date of death or the date an individual first developed an infection of interest. End of follow-up for unexposed individuals will also occur if IBD is diagnosed, at which point they become eligible for the exposed group. All individuals contributing $\geq 1$ day of follow-up time will be included in the analysis.

\section{Outcomes}

\section{Baseline measures}

Baseline measures include sociodemographic characteristics, BMI, smoking status and alcohol use, comorbidities, lymphocyte and neutrophil count (for the infections analysis) and IBD medication use. Socioeconomic status will be defined using the official national measure, the index of multiple deprivation, calculated at the point of data extraction, using patient postcode, with the resultant scores stratified by deprivation quintile. Ethnicity will be extracted from the primary care record and grouped into major ethnic groups: white, black, Asian, mixed and others. ${ }^{24} \mathrm{BMI}$ is defined as the most recently recorded measurement prior to the study start date. Smoking status and alcohol use are defined using the most recently recorded data prior to the study start date.

Diagnostic codes will be used to define the presence of comorbidities at baseline: diabetes mellitus, hypertension, hyperlipidaemia, atrial fibrillation, angina, myocardial infarction, congestive heart failure, peripheral vascular disease, stroke, transient ischaemic attack, chronic kidney disease stages 3-5, dementia, rheumatoid arthritis, chronic liver disease, asthma,
Table 1 Medication class groupings for treatment of IBD and mental health conditions

\begin{tabular}{|c|c|}
\hline Medication class & Medications \\
\hline \multicolumn{2}{|l|}{ Medication for IBD } \\
\hline Topical 5-ASA & Mesalazine, sulfasalazine \\
\hline Topical glucocorticoids & Budesonide, hydrocortisone, prednisolone \\
\hline Oral 5-ASA & Balsalazide, mesalazine, olsalazine, sulfasalazine \\
\hline Oral glucocorticoids & $\begin{array}{l}\text { Prednisolone, cortisone, dexamethasone, } \\
\text { methylprednisolone, beclomethasone, betamethasone, } \\
\text { budesonide, deflazacort }\end{array}$ \\
\hline Antimetabolites & Azathioprine, mercaptopurine, methotrexate \\
\hline Biological therapies & Adalimumab, golimumab, infliximab, vedolizumab \\
\hline \multicolumn{2}{|c|}{ Medications for mental health conditions } \\
\hline $\begin{array}{l}\text { SSRIs and related } \\
\text { medication }\end{array}$ & $\begin{array}{l}\text { Venlafaxine, bupropion, duloxetine, naltrexone, } \\
\text { citalopram, dapoxetine, escitalopram, fluoxetine, } \\
\text { fluvoxamine, paroxetine, sertraline }\end{array}$ \\
\hline TCAs & $\begin{array}{l}\text { Amitriptyline, clomipramine, dosulepin, doxepin, } \\
\text { mianserin, trazodone, trimipramine, imipramine, } \\
\text { lofepramine, nortriptyline }\end{array}$ \\
\hline MAOls & $\begin{array}{l}\text { Tranylcypromine, phenelzine, isocarboxazid, } \\
\text { moclobemide }\end{array}$ \\
\hline Anxiolytic medications & $\begin{array}{l}\text { Alprazolam, chlordiazepoxide, clobazam, clonazepam, } \\
\text { diazepam, lorazepam, oxazepam, buspirone, } \\
\text { meprobamate, clomipramine, clomethiazole }\end{array}$ \\
\hline
\end{tabular}

5-ASA, 5-aminosalicylic acid; IBD, inflammatory bowel disease; MAOI, monoamine oxidase inhibitors; SSRI, selective serotonin reuptake inhibitors; TCA, tricyclic antidepressants.

chronic obstructive pulmonary disease, joint replacement and fractures.

Medications for the treatment of IBD will be described: rectal 5-aminosalicylic acid (5-ASA) medications, rectal glucocorticoids, oral 5-ASA medications, oral glucocorticoids, nonbiological immunosuppressant medications and biological therapies (table 1). Medication use at baseline is defined as the issue of a prescription 3 months before, to 30 days after, the study start date.

\section{Infections outcomes (Study 1)}

Infection outcomes are: (1) the incidence of a new presentation of any common infection during the study period, (2) the incidence of a new presentation of viral infection and (3) the incidence of a new presentation of GI infection. Common infection was defined a priori as a composite of upper respiratory tract infections, pneumonia, acute bronchitis, influenza and influenza-like illness (ILI), skin infections, herpes simplex and herpes zoster infections, genital infections, urinary tract infections and GI infections. Each component infection will also be analysed separately.

The Read codes used to identify common infections are taken from the validated indicators used in routine surveillance by the RCGP RSC. Viral infections are defined as a composite of ILI, herpes simplex and herpes zoster infections and any upper or lower respiratory tract infections specifically coded as being viral in nature. GI infections comprise clinical diagnoses of gastroenteritis, enteritis or infective colitis, as well as laboratory confirmed viral, bacterial or protozoan GI tract infections.

In a secondary analysis, we will examine the incidence of all infections comprising the primary outcome of common infection in separate analysis and a subset of GI infections (a composite of organism-specified infections; Clostridium difficile, salmonella, shigella and campylobacter infections) as a sensitivity analysis. First or new presentations of an infection are coded accordingly 
in the database, enabling differentiation from chronic infections or follow-up visits for the same episode.

\section{Mental health outcomes (Study 2)}

The presence of mental health conditions at baseline will be determined for IBD and controls; three groups of mental health problems will be described, as defined by the International Statistical Classification of Diseases and Related Health Problems 10th Revision classification; depressive episodes (F32), recurrent depressive disorder (F33) and non-phobia related anxiety disorders (F41). ${ }^{25}$ These conditions represent the most common mental health conditions presenting to primary care and will be identified using algorithms validated in UK primary care. ${ }^{26} 27$ The algorithms selected optimise the positive predictive value and use a concurrent diagnosis and treatment code to identify acute episodes (depressive episodes and anxiety episodes) and for recurrent depression, either a historical code for any depression and current treatment or a recurrent depression-specific diagnosis code (online supplementary file 2).

\section{Healthcare use outcomes (Study 2)}

Healthcare use will be described: the number of primary care attendances, recorded emergency secondary care attendances and fitness for work notes; the number of primary care prescriptions for antidepressant and anxiolytic medications and in the IBD cohort only, the number of primary care prescriptions for IBDrelated medications. Medications evaluated for the treatment of IBD are topical 5-ASA medications, topical glucocorticoids, oral 5-ASA medications, oral glucocorticoids, non-biological immunosuppressant medications (cyclosporine, azathioprine, mercaptopurine, methotrexate) and biological therapies (table 1).

We will also explore the use of medications for mental health conditions; antidepressant and anxiolytic medications (table 1). Antidepressant medications are selective serotonin reuptake inhibitors and related medications (serotonin and norepinephrine reuptake inhibitors). Anxiolytic medications are all benzodiazepines and other related medications indicated for use in anxiety states.

\section{Statistical analyses \\ Infections (Study 1)}

Baseline clinical characteristics and outcome data will be described in individuals with IBD overall and separately in those with CD and UC and in the matched control population. Differences in baseline characteristics between the exposed and unexposed cohorts will be evaluated using the $\chi^{2}$ test for categorical variables and the unpaired t-test for continuous data.

\section{Risk of infection in IBD}

Incident cases among individuals with a first diagnostic Read code for infection during the study period will be identified and the incidence of common, GI and viral infections calculated for people with UC and CD (divide the number of incident patients by the sum of person-years of follow-up for the total eligible population over the study period; expressed as the number per 100 person-years).

The risk of presentation with infection in UC and CD will be estimated using an unadjusted Cox proportional hazards models, stratified by matched set, to provide overall HRs for the association with each infection outcome. Models will be subsequently adjusted for baseline measures as described above, using multivariable analysis.
Factors associated with infection in IBD

Baseline factors associated with an increased risk of presentation with infection will be evaluated in UC and CD separately, compared with controls, using Cox proportional hazards models; factors evaluated are age, sex, ethnicity, socioeconomic status, lifestyle factors (BMI, smoking, alcohol use), comorbidities, duration of disease and baseline medication use.

The time-varying impact of lymphopaenia and neutropenia over follow-up on the risk of presentation of infection will be explored using unadjusted and adjusted Cox proportional hazards models, with the current laboratory test results modelled as a time-varying covariate. Both continuous and categorical measures of lymphopaenia and neutropenia will be examined. Test results recorded in the 2 weeks prior to an infection will be excluded to reduce the likelihood the infection itself influenced the test result. In a sensitivity analysis, the exclusion window will be extended to the 2 months before an infection.

\section{Mental health conditions (Study 2)}

The prevalence of the three common mental health conditions in IBD and controls will be estimated at baseline, stratified by condition (UC or CD) and by age, sex, duration of disease and the use of systemic therapy. We will explore the impact of disease severity on outcomes with the use of systemic therapy as a surrogate marker and defined as the use of immunomodulating medications (mercaptopurine, azathioprine, cyclosporine, methotrexate or tacrolimus) or biological therapies.

\section{Healthcare use (Study 2)}

Measures of healthcare use will be assessed in patients with IBD and controls over the 3-year study period, reported as annual rates, calculated as the number of events (including multiple events per individual) divided by the total person-years of follow-up. Each individual will contribute person-time from baseline to the earliest of the study end-date (1 January 2019), the date of patient transfer from an included practice or date of death.

In both UC and CD, associations between anxiety episodes, depressive episodes and recurrent depression and the healthcare use outcome measures (primary care visit frequency, emergency hospital attendances and statements of fitness for work) will be assessed using multivariable generalised linear models such as Poisson regression. In addition to the presence or absence of each mental health condition, the multivariable feature set will comprise age category (years: 18-29, 30-39, 40-49, 50-59, 60-69, 70-79, 80+), sex, BMI category, duration of disease, socioeconomic status and smoking status. For BMI, socioeconomic status and lifestyle factors, the missing indicator variable method will be used to ensure all individuals were included in the models; as these data are likely to be missing not at random, multiple imputation approaches are likely to lack validity .

All statistical analyses will be performed in $\mathrm{R}$ statistical package 3.4.1.

\section{DISCUSSION}

\section{Strengths and limitations of the research methods}

Strengths include the use of RCGP RSC, the primary infectious disease sentinel network for the UK and the use of validated algorithms to define both IBD and the study outcomes of common infections and mental health conditions. While the network currently only covers England, the populationbased sample will be broadly representative of the UK, so our findings should have wider validity. The large sample size 
covered by the RCGP RSC network, and general practice level matching of cases and controls are additional strengths of our approach.

Limitations include the possibility that people with IBD have increased contact with healthcare providers, resulting in recording and data capture bias. As the data are observational in nature and the possibility of such residual confounding cannot be excluded, meaning results will not have a causal interpretation. ${ }^{6}$ In the UK, prescriptions for biological therapies are provided from secondary care and so may not be captured in the primary care record; therefore, our study is likely to systemically undercapture the prescribing of biological therapies. This is a problem common to all studies using UK electronic health records; many hospitals are still using handwritten prescriptions and therefore secondary care patient-level prescribing information is not currently captured electronically. A related point is the use of immunomodulating medications and biological therapies as markers of IBD disease severity; while these are the best markers we have for severe disease course, they may not reflect disease activity as it is possible for people with severe disease to have inactive disease-severity and activity are two related but different measures. Reliably assessing IBD disease activity accurately would be of considerable interest but any attempt to capture disease activity accurately would require validation studies that are beyond the scope of the proposed work. Surgery, another potentially useful marker of IBD disease severity, is not robustly captured in the primary care record and so was not used for this study.

A further limitation is the lack of secondary care data linkage on infections; although secondary care infection diagnoses are often transferred to primary care records, we are likely to systematically under capture infection diagnoses made in secondary care. However, RCGP RSC network has been the primary infections surveillance network for the UK for over 50 years and, through regular feedback to practices, it provides one of the highest quality real-world data samples capturing infectious diseases.

\section{Summary}

In conclusion, these studies will quantify any increased infection risk in people with IBD and identify patient characteristics associated with common infections in this group. It will also enable us to quantify the excess burden of anxiety and depression in a population-based IBD cohort compared with matched controls, and the impact of these conditions on healthcare use and time off work. A greater understanding and awareness of infection risk and common mental health issues is worthwhile for patients, doctors and policy makers. If our study demonstrates that people with IBD are at significantly increased infection risk, then patient and healthcare provider education could enable early recognition and treatment. This is particularly important in this group of people who are frequently treated with immunomodulating therapies. Studies similar to the mental health component of our study, in other chronic health conditions such as diabetes and psoriasis have resulted in increased recognition of the interrelationship between mental and physical health problems in these conditions and have led to substantial changes to treatment recommendations and policy. ${ }^{29}{ }^{30}$ If our study demonstrates an increased burden of mental health conditions in people with IBD, this will help to guide clinicians in screening for these problems (in both primary and secondary care) and support policymakers in allocating the right resources, following the models that have been used in other chronic diseases.
Acknowledgements Medical writing support was provided by John Dennis, Andrew McGovern and Annick Moon at Momentum Data and was funded by Pfizer. Project Management Support was provided by Filipa Ferreira at Oxford University and the University of Surrey.

Contributors All authors made substantial contributions. KB, PI, DT, MN and SdL designed the study, performed the data interpretation, supervised the writing of the statistical analysis and writing of the manuscript and performed a critical revision of the manuscript for important intellectual content. All authors have read and approved the final manuscript.

Funding This study was sponsored by Pfizer.

Competing interests This study is funded by Pfizer Ltd. KB has received honoraria from Tillots, Thermo Fisher Scientific, Boeringer Ingelheim, Pfizer and Yakult. PI has received lecture fees from AbbVie, Celgene, Falk Pharma, Ferring, MSD, Janssen, Pfizer, Takeda, Tillotts, Sapphire Medical, Sandoz, Shire and Warner Chilcott; financial support for research from MSD, Pfizer and Takeda; advisory fees from AbbVie, Arena, Genentech, Gilead, Hospira, Janssen, Lilly, MSD, Pfizer, Pharmacosmos, Prometheus, Roche, Sandoz, Samsung Bioepis, Takeda, Topivert, VH2, Vifor Pharma, and Warner Chilcott. DT and MN are employees of Pfizer. SdL is Director of RCGP RSC, and he had received funding for projects from Eli Lilly, Astra Zeneca, GSK, Seqirus and Takeda — all through his Universities and none related to this study.

\section{Patient consent for publication Not required.}

Ethics approval Study approval has been granted by the RCGP RSC Study Approvals Committee. The study does not meet the requirements for formal ethics board review as defined using the National Health Service (NHS) Health Research Authority research decision tool (http://www.hra-decisiontools.org.uk/research/). The planned studies are observational studies using the anonymised, routinely collected, data of primary care patients in the UK. This means there is no randomisation and there is no change to treatment or patient care as part of this study. As such explicit written or verbal consent is not required. All primary care practices providing data display messages to their patients informing them that their anonymised data may be used for observational research studies. All patients registered with participating centres are provided with the option to opt out of data sharing for this purpose. Data from patients choosing to opt out of data sharing are not analysed. All patient data are pseudonymised at the point of data extraction for the participating practices. Prior to inclusion in the planned studies data will be fully anonymised by the data custodian so no patient identifiable data will be available to researchers.

Provenance and peer review Not commissioned; externally peer reviewed.

Data availability statement Data may be obtained from a third party and are not publicly available. The RCGP RSC dataset is held securely at Oxford University and the University of Surrey and can be accessed by bone fide researchers. Approval is on a project-by-project basis (www.rcgp.org.uk/rsc). Ethical approval by an NHS Research Ethics Committee may be needed before any data release/other appropriate approval. Researchers wishing to directly analyse the patient-level pseudonymised data will be required to complete information governance training and work on the data from university secure servers. Patient-level data cannot be taken out of the secure network.

Open access This is an open access article distributed in accordance with the Creative Commons Attribution Non Commercial (CC BY-NC 4.0) license, which permits others to distribute, remix, adapt, build upon this work non-commercially, and license their derivative works on different terms, provided the original work is properly cited, appropriate credit is given, any changes made indicated, and the use is non-commercial. See: http://creativecommons.org/licenses/by-nc/4.0/.

\section{ORCID iD}

Simon de Lusignan http://orcid.org/0000-0002-8553-2641

\section{REFERENCES}

1 Abraham C, Cho JH. Inflammatory bowel disease. N Engl J Med 2009;361:2066-78.

2 Baumgart DC, Sandborn WJ. Crohn's disease. Lancet 2012;380:1590-605.

3 Ordás I, Eckmann L, Talamini M, et al. Ulcerative colitis. Lancet 2012;380:1606-19.

4 Catalan-Serra I, Brenna Øystein. Immunotherapy in inflammatory bowel disease: novel and emerging treatments. Hum Vaccin Immunother 2018;21:1-15.

5 Rahier JF, Magro F, Abreu C, et al. Second European evidence-based consensus on the prevention, diagnosis and management of opportunistic infections in inflammatory bowel disease. J Crohns Colitis 2014;8:443-68.

6 Toruner M, Loftus EV, Harmsen WS, et al. Risk factors for opportunistic infections in patients with inflammatory bowel disease. Gastroenterology 2008;134:929-36.

7 Gupta G, Lautenbach E, Lewis JD. Incidence and risk factors for herpes zoster among patients with inflammatory bowel disease. Clin Gastroenterol Hepatol 2006;4:1483-90.

8 Ford AC, Peyrin-Biroulet L. Opportunistic infections with anti-tumor necrosis factor- $\alpha$ therapy in inflammatory bowel disease: meta-analysis of randomized controlled trials. Am J Gastroenterol 2013;108:1268-76. 
9 Vögelin M, Biedermann L, Frei P, et al. The impact of Azathioprine-Associated lymphopenia on the onset of opportunistic infections in patients with inflammatory bowel disease. PLoS One 2016;11:e0155218.

10 Neuendorf R, Harding A, Stello N, et al. Depression and anxiety in patients with inflammatory bowel disease: a systematic review. J Psychosom Res 2016;87:70-80.

11 Thavamani A, Umapathi KK, Khatana J, et al. Burden of psychiatric disorders among pediatric and young adults with inflammatory bowel disease: a population-based analysis. Pediatr Gastroenterol Hepatol Nutr 2019:22:527-35.

12 Bounthavong M, Li M, Watanabe JH. An evaluation of health care expenditures in Crohn's disease using the United States medical expenditure panel survey from 2003 to 2013. Res Social Adm Pharm 2017:13:530-8.

13 Gracie DJ, Hamlin JP, Ford AC. Longitudinal impact of IBS-type symptoms on disease activity, healthcare utilization, psychological health, and quality of life in inflammatory bowel disease. Am J Gastroenterol 2018;113:702-12.

14 Kawalec P. Indirect costs of inflammatory bowel diseases: Crohn's disease and ulcerative colitis. A systematic review. Arch Med Sci 2016;12:295-302.

15 Lo B, Vind I, Vester-Andersen MK, et al. Direct and indirect costs of inflammatory bowel disease: ten years of follow-up in a Danish population-based inception cohort. J Crohns Colitis 2020;14:53-63.

16 Correa A, Hinton W, McGovern A, et al. Royal College of general practitioners research and surveillance centre (RCGP RSC) sentinel network: a cohort profile. BMJ Open 2016;6:e011092.

17 de Lusignan S, van Weel C. The use of routinely collected computer data for research in primary care: opportunities and challenges. Fam Pract 2006;23:253-63.

18 Kumar S, de Lusignan S, McGovern A, et al. Ischaemic stroke, haemorrhage, and mortality in older patients with chronic kidney disease newly started on anticoagulation for atrial fibrillation: a population based study from UK primary care. BMJ 2018;360:k342.

19 Nikiphorou E, de Lusignan S, Mallen C, et al. Haematological abnormalities in newonset rheumatoid arthritis and risk of common infections: a population-based study. Rheumatology 2020;59:997-1005.
20 Pathirannehelage S, Kumarapeli P, Byford R, et al. Uptake of a Dashboard designed to give RealTime feedback to a sentinel network about key data required for influenza vaccine effectiveness studies. Stud Health Technol Inform 2018;247:161-5.

21 Lewis JD, Brensinger C, Bilker WB, et al. Validity and completeness of the general practice research database for studies of inflammatory bowel disease. Pharmacoepidemiol Drug Saf 2002;11:211-8.

22 Abrahami D, Douros A, Yin H, et al. Dipeptidyl peptidase-4 inhibitors and incidence of inflammatory bowel disease among patients with type 2 diabetes: population based cohort study. BMJ 2018;360:k872.

23 Ananthakrishnan AN, Cai T, Savova G, et al. Improving case definition of Crohn's disease and ulcerative colitis in electronic medical records using natural language processing: a novel informatics approach. Inflamm Bowel Dis 2013;19:1411-20.

24 Tippu Z, Correa A, Liyanage H, et al. Ethnicity recording in primary care computerised medical record systems: an ontological approach. J Innov Health Inform 2017;23:799.

25 World Health Organization. International statistical classification of diseases and related health problems 10th revision, 2016. Available: http://apps.who.int/ classifications/icd10/browse/2016/en [Accessed Jan 2020].

26 Kessler RC, Chiu WT, Demler O, et al. Prevalence, severity, and comorbidity of 12-month DSM-IV disorders in the National comorbidity survey replication. Arch Gen Psychiatry 2005;62:617-27.

27 John A, McGregor J, Fone D, et al. Case-Finding for common mental disorders of anxiety and depression in primary care: an external validation of routinely collected data. BMC Med Inform Decis Mak 2016;16:35.

28 Pagoni P, Dimou NL, Murphy N, et al. Using Mendelian randomisation to assess causality in observational studies. Evid Based Ment Health 2019;22:67-71.

29 All Party parliamentary group for diabetes: diabetes and mental health. Available: https://diabetesappg.files.wordpress.com/2018/08/appg-mental-health-report_v3.pdf

30 National Clinical Guideline Centre (UK). Assessment and management of psoriasis, 2012. Available: https://www.nice.org.uk/guidance/cg153/ 